\title{
DETERMINACIÓN DE INTERVALOS DE REFERENCIA DE HEMOGLOBINA Y HEMATOCRITO EN UNA POBLACIÓN DE ESTUDIANTES DE ENTRE 18 Y 25 AÑOS DE AMBOS SEXOS DE LIMA, DE ACUERDO AL MÉTODO NO PARAMÉTRICO RECOMENDADO POR EL CLINICAL AND LABORATORY STANDARS INSTITUTE (CLSI), GUÍA C28-A3
}

\author{
DETERMINATION OF REFERENCE INTERVALS \\ OF HEMOGLOBIN AND HEMATOCRIT IN A POPULATION \\ OF STUDENTS BETWEEN 18 AND 25 YEARS OLD OF BOTH \\ GENDERS IN LIMA ACCORDING TO THE NONPARAMETRIC \\ METHOD RECOMMENDED BY THE CLINICAL AND \\ LABORATORY STANDARS INSTITUTE (CLSI), GUIDE C28-A3
}

PEDRO MENGOLÉ AMAYA

Universidad Norbert Wiener

\section{RESUMEN}

El objetivo del presente trabajo consiste en determinar los intervalos de referencia para hemoglobina y hematocrito en una población adulta-joven del departamento de Lima. Se siguieron los lineamientos de la guía C 28-A3 del CLSI, para lo cual se eligió como población de referencia a estudiantes de una institución de educación superior que superaron los criterios de exclusión fijados y accedieron ala extracción de sangre. Fueron tomadas muestras de 120 varones y 120 mujeres. Los analitos se determinaron por el método de cianmetahemoglobina y microcentrifugación, siguiendo procedimientos estandarizados en las guías CLSI H 15-A3 y H 07-A3 respectivamente. El intervalo de referencia se calculó por el método no paramétrico descrito en la guíaCLSIEP 38-A3.Los límites obtenidos fueron: hemoglobina=14$16.8 \mathrm{~g} / \mathrm{dl}$ (hombres), $11.3-14.3 \mathrm{~g} / \mathrm{dl}$ (mujeres) y hematocrito $=41-53 \%$ (hombres), $37-47 \%$ (mujeres). Estos resultados fueron comparados con otros valores obtenidos en diferentes estudios.

Palabras clave: intervalos de referencia, hemoglobina, hematocrito, población adulta joven de Lima, CLSI C 28-A3.

\section{ABSTRACT}

fte aim of the present work is to determine the reference intervals for Hemoglobin and Hematocrit in the young adult population of Lima. fte guidelines of the CLSI C 28-A3 document were followed, for which students from the Higher education educational institution were chosen as the reference population, who exceeded the exclusion criteria established and agreed to the blood collection. Two samples of 120 students each, between men and women, were selected. fte analytes were dosed by the cyanmetahemoglobin method and microcentrifugation following standardized procedures. fte reference interval was calculated by the nonparametric method and the limits obtained were: Hemoglobin $=14-16.8 \mathrm{~g} / \mathrm{dl}$ (men), 11.3-14.3 g / dl (women) and Hematocrit $=41-53 \%$ (men), 37-47 \% (women). ftese results were comparable to other values obtained in differentstudies.

Keywords: Referenceintervals, Hemoglobin, Hematocrit, young adult population of Lima, CLSI C 28-A3. 


\section{INTRODUCCIÓN}

\section{Antecedentes}

Determinar o verificar intervalos de referencia biológicos es parte fundamental del proceso de verificación de procedimientos de medida en el laboratorio; sin embargo, por mucho tiempo no ha existido una guía que uniforme criterios para lograr este objetivo. Appold (2009) señalaen sueditorial que «al emplearlasestrategias presentadas en la guía C28-A3, el laboratorio puede aumentar la confianza de los intervalos de referencia que reporta en susresultados». Indica además que la nueva versión dela guía permite validar intervalos de referencia, inclusive en laboratorios pequeños, usando solo 20 muestras de individuos de referencia sanos ${ }^{1}$.

La importancia de realizar este protocoloradica en que los valores que consideramos como "normales" para determinado analito, en realidad dependen de la población de estudio y no necesariamente serán los mismos que los descritos en la bibliografía. Brewsteretal.(2007), en su estudio sobre la distribución de Creatin Kinasa en la población general, descubrieron que los valores de referencia de la prueba declarados por el fabricante de un reactivo se encontraban de tres a cinco veces por debajo de los valores obtenidos en la población de estudio: 1500 individuos clasificados como sanos ${ }^{2}$.

Los rangos de referencia ayudan a describir lo que es más característico para un grupo particular de individuos en función de su edad, sexo u otras características. Galvis et al. (2016) estimaron los intervalos de referencia del perfil lipídico en una población atendida en un laboratorio, estableciendo que la importancia para el diseño de estrategias de prevención primaria para dislipidemias es la población estadística³ .

En Latinoamérica se observan pocos antecedentes de este tipo de estudio,Fernández et al. (2006) determinaron intervalos de referencia para los parámetros del autoanalizador hematológico GEN-S en una población de donantes voluntarios de sangre de la zona metropolitana de Caracas, Venezuela, y los intervalos hallados fueron similares a los reportados por la mayoría de autores comúnmente consultados en la literatura ${ }^{4}$. Sin embargo, a nivel nacional aún no se han estudiado valores de referencia para estos parámetros hematológicos. En ese contexto, uno de los objetivos del presente trabajo es establecer los rangos de referencia de una población local para los parámetros de hematocrito y hemoglobina.

Rosales Rimache et al. (2012) determinaron la prevalencia de anemia en estudiantesingresantesala Universidad Nacional Mayor de San Marcos en Lima, Perú, y destacaron la necesidad de contar con intervalos de referencia locales de hemoglobina para poder clasificar la presencia o ausencia de anemia, ya que, dependiendo de esa variable, la tasa de la enfermedad en la población evaluada puede variar considerablemente ${ }^{5}$, información que este trabajo puede proveer.

Sin embargo, cabe resaltar que los rangos de referencia determinados en este estudio son válidos para poblaciones con características similares a las de la población de estudio. Toledo Jaramillo (2010) determinó valores de referencia de hemoglobina en una población estudiantil masculina de 12 a 19 años en Loja, Ecuador, y concluyó que el intervalo hallado de 12.8-17.2 g/ $\mathrm{dl}$, con un promedio de $15 \mathrm{~g} / \mathrm{dl}$, denotaba 
poca variación con respecto a otros estudios realizados en lugares de similar geografía y población ${ }^{6}$.

\section{Objetivos}

\section{General}

Establecer los rangos de referencia de una población local para los parámetros de hemoglobina y hematocrito.

\section{Específicos}

- Estandarizar los procesos de preanálisis de hemoglobina y hematocrito según las normas CLSI H7-A3 y H15-A3.

- Estandarizar los procesos para el análisis de hemoglobina y hematocrito según las normas CLSI H7-A3 y H15-A3.

- Establecer los rangos de referencia locales para hemoglobina según sexo.

- Establecer los rangos de referencia locales para hematocrito según sexo.

\section{MATERIALESY MÉTODOS}

\section{Metodología}

Se realizó un estudio prospectivo de tipo experimental entre los meses de abril y mayo de 2018. La muestra deestudio estuvo conformada por 240 pacientes, 120 varones y 120 mujeres, de acuerdo a los lineamientos del protocolo CLSI C28-A3.

Los valores de referencia fueron obtenidos de una población clínicamente sana y homogénea. Se seleccionó en función de los resultados de las encuestas de inclusión-exclusión aplicadas a los potenciales participantes.

\section{Muestreo}

Población de referencia. Se recolectaron 240 muestras de pacientes seleccionados por conveniencia, que acudieron al chequeo preventivo (hombres y mujeres). Estos aceptaron participaren elestudioy completaron el consentimiento informado y un cuestionario validado e incluido en el protocolo CLSI C 28-A37.

Criterios de inclusión. Se consideraron los siguientes: mayores de 18 años, clínicamente sanos (diagnóstico descrito en la historia clínica según su evaluaciónmédica y cuestionario validado), que no ingirieron alcohol el día anterior y se encontraban en ayuno de 12 horas.

\section{Pruebas de laboratorio}

Toma de muestra y procesamiento. Las muestras de sangre venosa fueron obtenidas por procedimientos de venopunción estándar ${ }^{8}$ en tubos al vacío con EDTA Tripotásico como anticoagulante y procesada dentro de las 2 horas posteriores.

Para hematocrito, las muestras fueron separadas en tubos capilares no heparinizados y centrifugadas a $11000 \mathrm{~g}$ por $5 \mathrm{mi}$ nutos, según lo descrito en la Norma CLSI H7-A $3^{9}$.

Parahemoglobina, se trabajaron muestras de sangre total de acuerdo a lo descrito en la Norma CLSI H15-A $3^{10}$ (20 ul de sangre total con $5.0 \mathrm{ml}$ de reactivo para hemoglobina). Previo a cada día de trabajo, se corrieron controles de calidadpara hemoglobina: niveles normal y patológico, alto y bajo. Se revisaron las gráficas de Levey Jennings y reglas deWestgard correspondientes para validar la corrida. 


\section{Análisis estadístico}

Se analizaron los datos para determinar la presencia de outliers por la prueba de Grubbs. Para el análisis estadístico se utilizó una hoja de cálculoExcel.

La prueba de Grubbs se basa en el supuesto de normalidad. Es decir, primero se debe verificar que los datos se puedan aproximar razonablemente mediante una distribución normal antes de aplicarla.

La prueba de Grubbs detecta un valor atípico a la vez. Este valor atípico se suprime del conjunto de datos y la prueba se repite hasta que no se detecten valores atípicos. Sin embargo, las iteraciones múltiples cambian las probabilidades de detección, y la prueba no debe usarse para tamaños de muestra de seis o menos, ya que con frecuencia etiquetala mayoría de los puntos como valores atípicos.
Es útil para tomar decisiones estadísticas en el rechazo de discrepantes, y se procede como sigue:

1. Se disponen los datos en orden de menor a mayor.

2. Se decide si el más pequeño o el más grande es sospechoso de ser discrepante.

3. Se estimala desviación estándar"s" de todos los datos.

4. Se seleccionael riesgoque sequieretomar para un falso rechazo.

5. Se calcula el valor correspondiente y se compara con el valor de tablas. Si el valor calculado es mayor que el valor tabulado, se puede rechazar ese dato con el riesgo asumido.

Los resultados obtenidos son los siguientes:

\section{Evaluación de datos aberrantes con filtro de Grubbs Resultado: no existen datos aberrantes u outliers}

\author{
Hemoglobina varones \\ Mean: 15.508 \\ SD: 0.830 \\ \# of values: 120 \\ \# of rows w/o data: 1 \\ Outlier detected?: No \\ Significance level: 0.05 (two-sided) \\ Critical value of Z: 3.44514951106
}

\section{Hematocrito varones}

$\begin{array}{rll}\text { Mean: } & 46.98 \\ \text { SD: } & 2.55 \\ \text { \# of values: } & 120 \\ \text { \# of rows w/o data: } & 1 \\ \text { Outlier detected?: } & \text { No } \\ \text { Significance level: } & 0.05 \text { (two-sided) } \\ \text { Critical value of Z: } & 3.44514951106\end{array}$




\section{Evaluación de datos aberrantes con filtro de Grubbs \\ Resultado: no existen datos aberrantes u outliers}

\author{
Hemoglobina mujeres \\ Mean: 12.671 \\ SD: $\quad 0.854$ \\ \# of values: 120 \\ \# of rows w/o data: 1 \\ Outlier detected?: No \\ Significance level: 0.05 (two-sided) \\ Critical value of Z: 3.44514951106
}

\author{
Hematocrito mujeres \\ Mean: 39.31 \\ SD: 2.11 \\ \# of values: 120 \\ \# of rows w/o data: 1 \\ Outlier detected?: No \\ Significance level: 0.05 (two-sided) \\ Critical value of $\mathrm{Z}: \quad 3.44514951106$
}

Para evaluar si los datos obtenidos presentan una distribución normal se aplicó el test de Shapiro-Wilk. La lógicade la prueba se basa en las desviaciones que presentan las estadísticas de orden dela muestra, respecto a los valores esperados de los estadísticos de orden de la normal estándar. Para efectuarla se calcula la media y la varianza muestral, S2, y se ordenan las observaciones de menor a mayor. A continuación, se calculan las diferencias entre el primero y el último, el segundo y el penúltimo, el tercero y el antepenúltimo, etc, y se corrigen concoeficientes tabulados por Shapiro y Wilk.

\section{RESULTADOS}

Fueron procesadas un total de 240 muestras de sangre de individuos aparentemente sanos con una edad comprendida entre 18 y 25 años, para determinar los intervalos de referencia para hemoglobina y hematocrito en el departamento de Lima.

Los intervalos de referencia fueron calculados siguiendo los criterios a priori del CLSI utilizando los percentiles 2,5\% y 97,5\%.Los valores finalmenteobtenidos aparecen en la Tabla 1.

TABLA 1

Valores de referencia obtenidos

\begin{tabular}{lcccc}
\hline Parámetros & \multicolumn{4}{c}{ Valores de Referencia IC 95 \% } \\
\hline & \multicolumn{3}{c}{ Mujeres } & \multicolumn{3}{c}{ Varones } \\
\cline { 2 - 5 } & Percentil 2,5 & Percentil 97,5 & Percentil 2,5 Percentil 97,5 \\
\cline { 2 - 5 } Hemoglobina (g/dl) & 11.3 & 14.6 & 14.1 & 16.8 \\
Hematocrito (\%) & 34 & 44 & 42 & 50 \\
\hline
\end{tabular}




\section{Hemoglobina en varones}

En la Figura 1 se muestra el histograma rencia estimado para hemoglobina fue de valores para hemoglobina en varones. tomado como el conjunto de valores El test de Shapiro-Wilk para evaluar nor- comprendido entre los percentiles 2,5 y malidad dio un $\mathrm{p}=0.051$ ( $\left.\mathrm{p}_{\text {esperado }}>0.05\right), \quad 97,5$ de la distribución y se muestra en la indicando que la distribución es razona- Tabla 1. El IC $95 \%$ quedó definido entre blemente normal. El intervalo de refe- $14.1 \mathrm{~g} / \mathrm{dL}$ y $16.8 \mathrm{~g} / \mathrm{dL}$.

\section{FIGURA 1}

Distribución de los valores de hemoglobina en varones

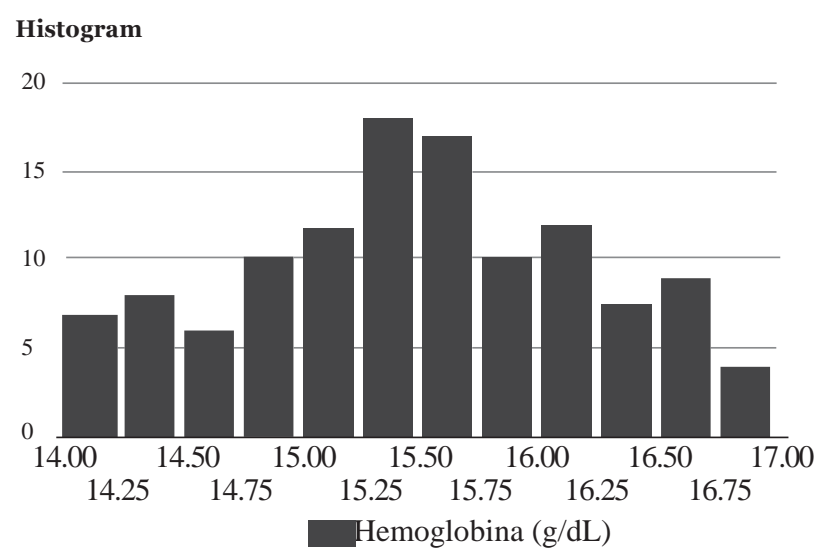

\section{Hematocrito en varones}

En la Figura 2 se muestra el histograma de valores para hematocrito en varones. El test de Shapiro-Wilkpara evaluar normalidad dio un $\mathrm{p}=0.052\left(\mathrm{p}_{\text {esperado }}>0.05\right)$, indicando que la distribución es razonablemente normal. El intervalo de referencia estimado para hematocrito fue tomado como el conjunto de valores comprendido entre los percentiles 2,5 y 97,5 de la distribución y se muestra en la Tabla 1. El IC 95\% quedó definido entre $42 \%$ y $50 \%$.

\section{FIGURA 2}

\section{Distribución de los valores de hematocrito en varones}

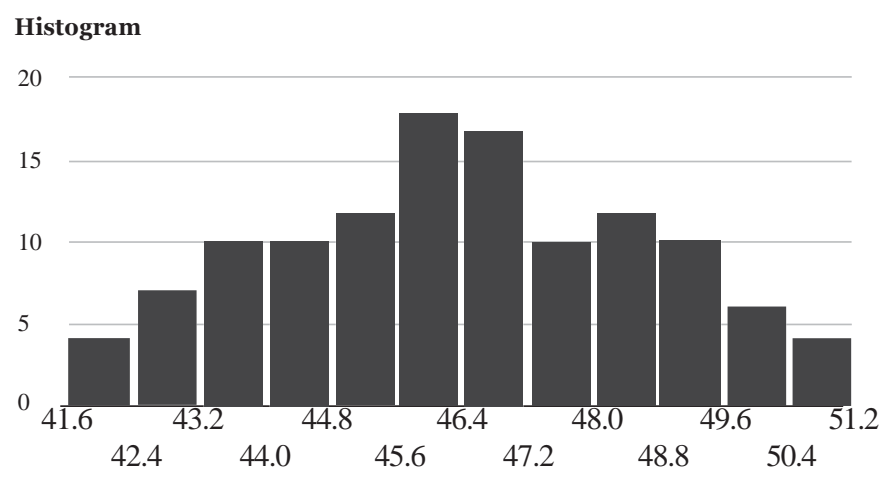


Hematocrito $(\%)$ 


\section{Hemoglobina en mujeres}

En la Figura 3 se muestra el histograma de valores para hemoglobina en mujeres. El test de Shapiro-Wilk para evaluar normalidad dio un $\mathrm{p}=0.077$ $\left(\mathrm{p}_{\text {esperado }}>0.05\right)$, indicando que la distribución es razonablemente normal.
El intervalo de referencia estimado para hemoglobina fue tomado como el conjunto de valores comprendido entre los percentiles 2,5 y 97,5 de la distribución y se muestra en la Tabla 1. El IC $95 \%$ quedó definido entre 11.3 $\mathrm{g} / \mathrm{dL}$ y $14.6 \mathrm{~g} / \mathrm{dL}$.

\section{FIGURA 3}

Distribución de los valores de hemoglobina en mujeres

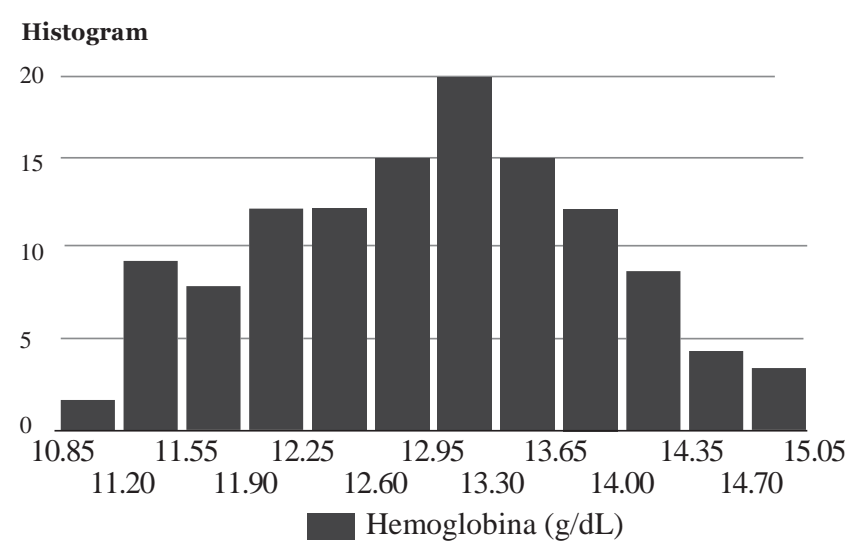

\section{Hematocrito en mujeres}

En la Figura 4 se muestra el histograma de valores para hematocrito en mujeres. El test de Shapiro-Wilk para evaluar normalidad dio un $\mathrm{p}=0.079$ ( $\mathrm{p}_{\text {esperado }}$ $>0.05$ ), indicando que la distribución es razonablemente normal. El intervalo de referencia estimado para hematocrito fue tomado como el conjunto de valores comprendido entre los percentiles $2,5 \mathrm{y}$ 97,5 de la distribución y se muestra en la Tabla 1. El IC $95 \%$ quedó definido entre $34 \%$ y $44 \%$.

\section{FIGURA 4}

Distribución de los valores de hematocrito en mujeres

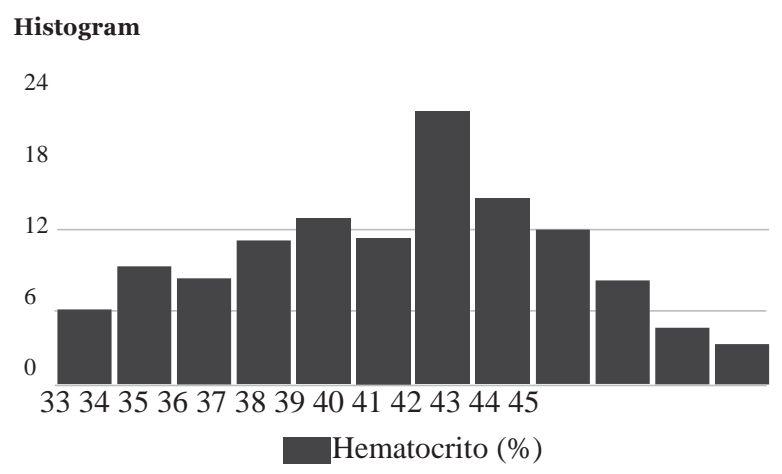




\section{DISCUSIÓN}

Es evidente la necesidad de los laboratorios clínicos de llevar adelante el procedimiento de verificación y/oestimación de intervalos de referencia de los analitos medidos. Esta circunstancia puede ocurrir por la veloz evolución de la metodología, por la incorporación de nuevos marcadores de relevancia clínica y, eventualmente, por el cambio en el perfil poblacional.

La obtención de valores de referencia para hemoglobina y hematocrito es un aporte importante que servirá como referencia para todas aquellas instituciones que requieran calcular sus propios intervalos de referencia, de conformidad con lo indicado en la NTP ISO 15189:2014 ${ }^{11}$, y también como dato de referencia para los investigadores que realicen estudios sobre prevalencia de anemia para clasificar adecuadamente a su población.

Si comparamos los resultados obtenidos (véase Tabla 1) con los obtenidos por otros autores (véase Tabla 2), se puede observar que son similares para los parámetros de hemoglobina y hematocrito, en varones y mujeres.

TABLA 2

Resumen de los valores de referencias reportados por diferentes autores

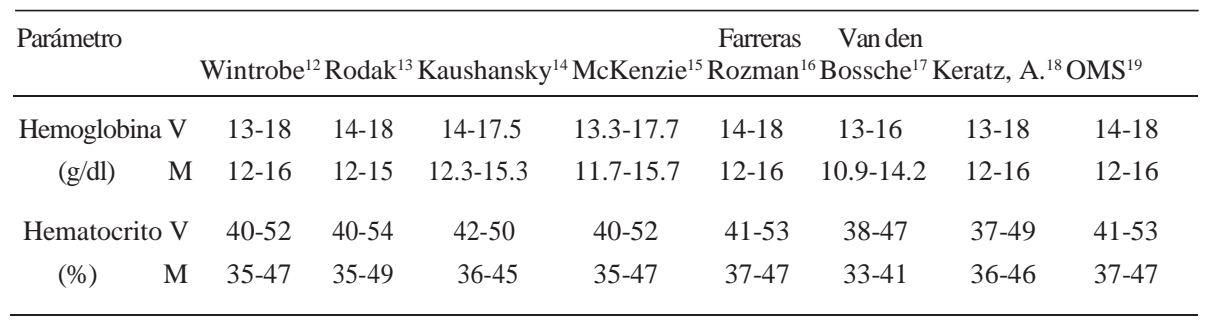

Tomando en consideración que los procedimientos para el análisis de las muestras fueron estandarizados según la guía GP41 del CLSI, y que se trabajaron controles diarios, se puede asegurar la validez de los resultados obtenidos en el estudio.

\section{V.CONCLUSIONES}

Se concluye que los valores de referencia hallados para hemoglobina y hematocrito en la población de estudio son las siguientes:

- Varones: 14.1-16.8 g/dL y 42-50 \%

- Mujeres: 11.3-14.6 g/dL y 34-44 \%
Los resultados que fueron considerados en el estudio fueron obtenidos utilizando protocolos internacionales estandarizados para la toma de muestra y procesamiento de hemoglobina y hematocrito, lo que asegura su validez.

Los resultados fueron filtrados y se evaluó su distribución normal para asegurar su consistencia. No se detectó ningún dato aberrante y el conjunto tuvo una distribución normal.

Los rangos de referencia hallados son similares a los descritos por otros autores en la literatura y pueden ser utilizados en estudios epidemiológicos realizados a ni- 
vel nacional en poblaciones con características similares alapoblación de estudio.

Los valores de referencia obtenidos pueden ser aplicados a poblaciones con características demográficas similares a las estudiadas, tal y como lo mencionan otros autores consultados. Es por esta razón que se observa la necesidad de realizar este tipo de estudio en diferentes zonas geográficas del país, cambiandolas variables de edad y de género, para obtener finalmente una base de datos de intervalos de referencia nacionales que pueda serutilizadaporotros investigadores y profesionales de la salud.

Nocabe duda que la correctainterpretación de un resultado depende en gran parte de intervalos de referencia verosímiles para el analito en cuestión. En este caso, el empleo de intervalos inadecuados para la metodología o para la población de pacientes, redundaba en la mala clasificación de algunos de ellos, sobre todo los que estaban próximos o excedían ligeramente el límite inferior, generando dudas en su interpretación y repeticiones probablemente innecesarias.

\section{Contribución al desarrollo del país}

En el Perú no existen actualmente antecedentes de trabajos similares, y anivel latinoamericano, existen escasos trabajos que propongan una metodología para el establecimiento de intervalos de referencia. Sin embargo, sí existen trabajos a nivel nacional que estudian la prevalencia de anemia utilizando valores de referencia internacionales para clasificar la presencia o ausencia de la enfermedad, lo que puede inducir a clasificaciones incorrectas ya que la población de estudio no es similar a la estudiada para obtener dichos intervalos. Los valores hallados en este trabajo pueden ser utilizados como referencia en este tipo de estudios, lo que ayudará en una mejor clasificación de los resultados.

Como una Universidad cuya visión es ser referenteen los sistemas y servicios de salud a nivel nacional, tenemos una gran oportunidad de formar parte de este nuevo paradigma enfocado en la calidad aportando nuevos conocimientos en un campo que aún no se ha explorado a fondo como el que propone éste proyecto.

Los resultados de este trabajo pueden ser aplicados y replicados por todos los laboratorios a nivel Nacional, lo que sería de gran ayuda para todos aquellos que busquen una acreditación nacional; ello, sumado a los esfuerzos del Inacal para estandarizar a los laboratorios clínicos puede posicionar a la Universidad como su aliado estratégico en la búsqueda de la calidad y estandarización en el Perú. 


\section{REFERENCIAS BIBLIOGRÁFICAS}

1. Appold. Determining Laboratory Reference Intervals: CLSI Guideline Makes the Task Manageable. Laboratory Medicine. 2009 February; 40(2): p. 75-76.

2. Brewster L, Gideon, Sturk A, van Montfrans GA. Distribution of creatine kinase in the general population: Implications for statin therapy. American Heart Journal. 2007 October; 154(4): p. 655-661.

3. Galvis, Barona J, Antoni. Intervalos biológicos de referencia del perfil lipídico. Acta Médica Colombia. 2016; 41(1): p. 29-35.

4. Fernández LE, Bustamante, García G. Valores de referencia obtenidos con el autoanalizador Coulter GEN-S. Revista de la Facultad de Medicina. 2006 Junio; 29(1).

5. Rosales Rimache JA, Alarcón Baldeón, Abadie Timaná JdM, Olivares Sánchez M. Prevalencia de anemia en estudiantes ingresantes a la Universidad Nacional Mayor de San Marcos del Perú. Boletín del Instituto Nacional de Salud. 2012 Julio-Agosto; 18(1).

6. Toledo Jaramillo. Valores referenciales de hemoglobina en la población estudiantil masculina de 12-19 años de los colegios fiscales de la ciudad de Loja. 2010.

7. Clinical and Laboratory Standars Institute. EP28-A3: Defining, Establishing, and Verifying Reference Intervals in the Clinical Laboratory. 3rd ed. Horowitz GL, editor. Pennsylvania: CLSI; 2010.
8. Clinical and Laboratory Standars Institute. GP41:Collection of Diagnos- tic Venous Blood Specimens. 7th ed. Erns DJ, editor. Pennsylvania: CLSI; 2017.

9. Clinical and Laboratory Standards Institute. H07-A3: Procedure for Determining Packed Cell Volume by the Microhematocrit Method. 3rd ed. Gottfried EL, Arkin CF, editors. Pennsylvania: CLSI; 2000.

10. Clinical and Laboratory Standards Institute. H15-A3:Reference and Selected Procedures for the Quantitative Determination of Hemoglobin in Blood. 3rd ed. Gottfried EL, Arkin CF, editors. Pennsylvania: CLSI; 2000.

11. INACAL. NTP-ISO 15189:2014 Laboratorios Clínicos. Requisitos particulares para la calidad y la competencia Lima: INACAL;2014.

12. Wintrobe M. Hematología Clínica Wintrobe. Novena ed. Buenos Ai- res: Inter-Médica; 1995.

13. Rodak BF, Fritsma GA, ME. Hematología: Fundamentos y aplicaciones clínicas. Cuarta ed. Buenos Aires: Editorial Médica Panamericana; 2014.

14. Kaushansky K, Lichtman M, Kipps T, Prchal J, Seligsohn U. Williams Hematology. 8th ed. New York: McGraw-Hill; 2002.

15. McKenzie S. Hematología Clínica. Segunda ed. Buenos Aires: El Manual Moderno; 2005.

16. Farreras Rozman. Medicina Interna. 14th ed. Madrid: Elsevier; 2000. 
17. Van den Bossche, Devreese K, Malfait, Van de Vyvere, Wauters, Neeis, et al. Reference intervals for a complete blood count determined on diffe- rent automated haematology analy- sers: Abx Pentra 120 Retic, Coulter Gen-S, Sysmex SE 9500, Abbott Cell Dyn 4000 and Bayer Advia 120. Clinical Chemestry and Laboratory

Medicine. 2002 January; 40(1).
18. Kratz A, Lewandrowski KB. Normal Reference Laboratory Values. fte New England Journal of Medicine. 1998 October; 339 (15).

19. Organización Mundial de la Salud. Concentraciones de hemoglobina para diagnosticar la anemia y evaluar su gravedad: OMS; 2011. 